
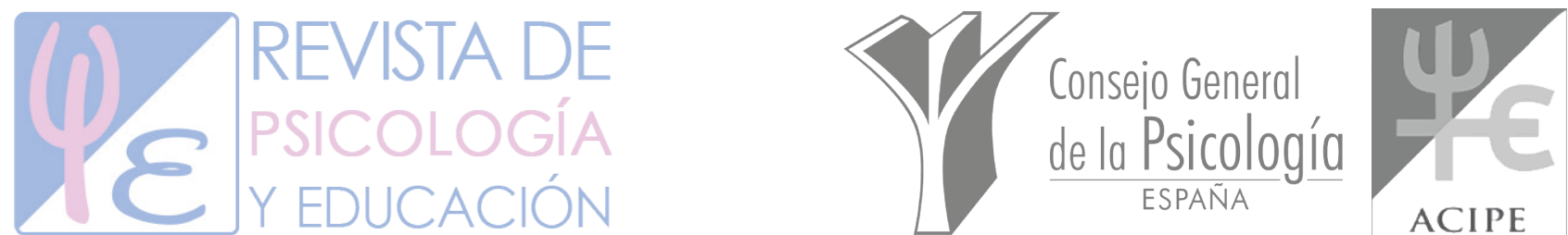

Revista de Psicología y Educación / Journal of Psychology and Education, 201 7, 12(2), 95-104 (www.rpye.es)

Doi: https://doi.org/10.23923/rpye2017.12.148

ISSN: 1699-9517

\title{
La violencia escolar en la Educación Secundaria Obligatoria desde la perspectiva del alumnado gallego
}

\author{
José Dominguez-Alonso ${ }^{*}$, Enrique Álvarez-Roales', Antonio López-Castedo' \\ Universidad de Vigo
}

\begin{abstract}
Resumen. La consolidación de la violencia en los centros educativos, ha suscitado gran preocupación en las comunidades educativas y científicas. Por ello, el objetivo principal del estudio es identificar los tipos de violencia más y menos habituales en la Educación Secundaria Obligatoria de Galicia, a partir de la percepción del alumnado. Se aplicó el cuestionario CUVE3-ESO (Álvarez-García, Núñez y Dobarro, 2012) a una muestra formada por 4943 adolescentes entre 12 y 17 años $(M=14.05 ; D T=1.39)$. Los resultados del análisis descriptivo muestran como tipos de violencia escolar percibidos más habituales: disrupción en el aula, violencia verbal entre estudiantes y violencia que ejerce el profesorado hacia el alumnado; y con menor asiduidad: exclusión social, violencia física indirecta y violencia a través de las nuevas tecnologías de la información y comunicación. En conclusión, las diferentes formas de violencia escolar sí a parecen en mayor o menor medida en los centros de educación secundaria obligatoria, aunque los valores encontrados en este estudio se mantienen en niveles bajos.
\end{abstract}

Palabras clave: Violencia Escolar, Educación Secundaria Obligatoria, Relación Profesorado-alumnado, Relación entre Escolares.

School violence in Galician compulsory secondary education from the perspective of students.

Abstract. The consolidation of violence in educational centers, has aroused a great concern in the educational and scientific communities. Therefore, the main objective of the study is to identify the types of violence more and less common in the compulsory secondary education in Galicia, from the perception of the students. The CUVE3-CSE questionnaire (Álvarez-García, Núñez \& Dobarro, 2012) was applied to a sample consisting of 4943 secondary students between 12 and 17 years $(M=14.05 ; D T=1.39)$. The results of the descriptive analysis show the most common types of school violence: disruption in the classroom, verbal violence between students and violence exercised by the teachers to the students; and with less assiduity: indirect physical violence, social exclusion and violence through the new information and communication technologies. In conclusion, the various forms of school violence do appear to a greater or lesser extent in compulsory secondary education centers, although the values found in this study remain low.

Keywords: School Violence, Compulsory Secondary Education, Teacher-student relationship, Relationship Between School.

La violencia escolar es un problema todavía muy presente en los centros educativos y requiere pues, de un mayor nivel de análisis y

Recibido: 12/04/2017 - Aceptado: 17/05/2017 - Avance online: 20/06/2017

*Correspondencia: José Dominguez Alonso

Facultad de Ciencias de la Educación (Campus de Ourense)

C.P: 32004, Ourense, España.

E-mail: jdalonso@uvigo.es

Dominguez, J., Álvarez, E. \& López, A. (2017). La violencia escolar en la Educación Secundaria Obligatoria desde la perspectiva del alumnado gallego. Revista de Psicología y Educación, 12(2), 95-104, https://doi.org/10.23923/rpye2017.12.148 profundidad (Romera, Del Rey y Ortega, 2011). No obstante, han sido muchas las iniciativas y esfuerzos llevados a cabo en la prevención e intervención sobre los problemas de violencia escolar (Álvarez-García, Dobarro, Álvarez, Núñez y Rodríguez, 2014; Cerezo y Calvo, 2011; Garaigordobil, 2009), registrando un importante avance y preocupación social en

1699-9517/@ 2017 Asociación Científica de Psicología y Educación (ACIPE). Publicado por Consejo General de Colegios Oficiales de Psicólogos, España. Este es un artículo Open Access bajo la CC BY-NC-ND licencia (http://creativecommons.org/licencias/by-nc-nd/4.0/). 
los últimos años (Garaigoldobil, MartínezValderrey y Machimbarrena, 2017; Palmero, Guerrero, Gómez, Carpi y Gorayeb, 2010)

La situación general de los centros educativos revela que se han hecho progresos sustanciales en el clima de convivencia escolar y se hace patente la incidencia positiva que han ejercido la multitud de estudios e investigaciones realizadas en este campo. No obstante, dado el dinamismo y la ubicuidad de la violencia en los espacios educativos, se crean nuevos desafíos que surgen de su realidad cotidiana y constituye un gran obstáculo para el logro de la excelencia educativa. Así, la necesidad de intensificar esfuerzos para reducir y prevenir la violencia es cada vez más acuciante. Para evaluar el impacto de la misma, es fundamental contar con mecanismos de seguimiento que permitan incorporar nuevas variables a los modelos explicativos existentes y así poder desarrollar herramientas de prevención e intervención que funcionen de enlace entre lo investigado y los centros educativos donde acontecen estas situaciones de violencia.

Así pues, ante las perspectivas actuales que reflejan la dificultad de definir y delimitar con precisión el término violencia escolar (AyalaCarrillo, 2015; Giorgi, Kaplún y Morás, 2012; Torrego, 2007), existe un cierto consenso en considerarla cómo cualquier tipo de comportamiento violento que se da en los centros educativos (Osorio, 2006; Serrano e Iborra, 2005). En consecuencia, se hace referencia a aquellas conductas intencionadas mediante las cuales se causa daño, ya sea por acción u omisión, pudiendo ser ejercida - padecida por cualquier miembro de la comunidad educativa (Guerra et al., 2011 ).

En consecuencia, la violencia en contextos escolares presenta una tipología amplia, variada y multidimensional, que constituye el fenómeno de la violencia escolar (Baridon y Martín, 2014; Hernández, Martínez y Torres, 2006). Por ello, es fundamental entender que la violencia en el ámbito escolar puede manifestar formas muy diversas, entre las que destaca la violencia física y verbal, la exclusión social, la disrupción en las aulas y la emergente violencia a través de las tecnologías de la información y comunicación (Álvarez-García, Rodríguez,
González-Castro, Núñez y Álvarez, 2010). Del mismo modo, Musri (2012) distingue entre exclusión y marginación social, violencia verbal y física (directa e indirecta), intimidación, amenaza o chantaje, violencia sexual, y ciberacoso. En resumen, la violencia escolar incluye múltiples causas (Arias, 2009; Steffgen, Recchia y Viechtbaver 2013), que abarcan desde actos delictivos leves hasta patrones de comportamiento más graves relacionados con agresiones físicas o verbales al profesorado o compañeros (Cava, Buelga, Musitu y Murgui, 2010; Díaz-Aguado, Martínez y Martín, 2004). El informe mundial sobre la violencia contra los niños (Pinheiro, 2011) define como principales formas de violencia: castigo físico y psicológico, acoso, violencia sexual o por razones de género y violencia externa (bandas, situaciones de conflicto, armas y peleas). Lo importante es destacar que ninguna de estas formas es causa de violencia escolar por sí sola (García, 2011).

De hecho, la violencia escolar es entendida como un fenómeno en el cual convergen elementos personales, grupales, sociales y culturales (Berger y Lisboa, 2009; Espelage y Swearer, 2010). Así pues, son numerosos los estudios que señalan como formas de violencia escolar más comunes en la educación secundaria obligatoria la agresión verbal indirecta y la exclusión social (Defensor del Pueblo, 2007; Martínez, Hernández-Aguado y Torres, 2006; Pagani et al., 2009). Asimismo, también son muchas las investigaciones que apuntan a la etapa de secundaria como la de mayor presencia de conductas violentas (Almeida, Lisboa y Caurcel, 2007; Félix, Soriano y Godoy, 2009; Muñoz, Pérez y Martín, 2006).

Por tanto, el interés por estudiar la violencia en contextos educativos está en constante crecimiento (Cabezas y Monge, 2013; Cajigas, Luzardo y Mungay, 2013; Martínez, Tovar, Rojas y Duque-Franco, 2008), registrando un claro avance en la última década como un fenómeno transversal en los centros de enseñanza. El objetivo de este trabajo es identificar los tipos de violencia más y menos habituales en la Educación Secundaria Obligatoria de Galicia, a partir de la percepción del alumnado. 


\section{MÉTODO}

\section{PARTICIPANTES}

La población objeto de estudio está constituida por la totalidad del alumnado que asiste a Educación Secundaria Obligatoria en Galicia (España), en centros sostenidos con fondos públicos (públicos y concertados). En el curso 2014/15 ascienden a un total de 86788 estudiantes. La muestra está compuesta por 4943 alumnos (Tabla 1), con edades comprendidas entre los 12 y 17 años $(M=14.05 ; D T=1.39)$. Como se puede observar en la Tabla 1, los porcentajes de estudiantes en la muestra en función del curso, el género y la titularidad del centro son muy similares a los de la población. La única divergencia destacable es una leve sobrerrepresentación de las chicas en la muestra, en comparación con la población.

\begin{tabular}{|c|c|c|c|c|}
\hline \multicolumn{5}{|c|}{$\begin{array}{c}\text { Tabla } 7 . \\
\text { Características de la muestra y de la población de estudiantes, en } \\
\text { función de las variables titularidad del centro, curso y género del } \\
\text { alumnado. }\end{array}$} \\
\hline & \multicolumn{2}{|c|}{ Muestra } & \multicolumn{2}{|c|}{ Población } \\
\hline & $\mathbf{N}$ & $\%$ & $\mathbf{N}$ & $\%$ \\
\hline Pública & 3512 & 71.0 & 62294 & 71.7 \\
\hline Concertada & 1431 & 29.0 & 24494 & 28.3 \\
\hline $1^{\circ} \mathrm{ESO}$ & 1336 & 27.0 & 23184 & 26.7 \\
\hline $2^{\circ} \mathrm{ESO}$ & 1291 & 26.1 & 23055 & 26.6 \\
\hline \multicolumn{5}{|l|}{ Curso } \\
\hline 3०ESO & 1262 & 25.5 & 21425 & 24.7 \\
\hline $4^{\circ} \mathrm{ESO}$ & 1054 & 21.3 & 19124 & 22.0 \\
\hline Alumnos & 2411 & 48.8 & 44682 & 51.5 \\
\hline \multicolumn{5}{|l|}{ Género } \\
\hline Alumnas & 2532 & 51.2 & 42106 & 48.5 \\
\hline Total & 4943 & 100 & 86788 & 100 \\
\hline
\end{tabular}

\section{INSTRUMENTO DE EVALUACIÓN}

Se aplicó el cuestionario CUVE3-ESO (Álvarez-García, Núñez, y Dobarro, 2012). Se trata de una prueba dirigida a estudiantes de ESO, mediante la cual se evalúa la frecuencia con la que consideran que ocurren diferentes tipos de violencia escolar protagonizados por el alumnado y el profesorado de su clase. Se trata de una escala tipo Likert de 44 enunciados, con cinco opciones de respuesta (desde 1 -Nuncahasta 5 -Siempre-). Presenta una estructura compuesta por ocho tipos de violencia escolar. La consistencia interna de cada factor en la muestra analizada en el presente trabajo es buena: Disrupción en el Aula ( $\alpha=.77)$; Violencia Verbal entre el Alumnado $(\alpha=.75)$; Violencia Física Directa y Amenazas entre Estudiantes $(\alpha=.81)$; Violencia Física Indirecta por parte del Alumnado ( $\alpha=.76)$; Exclusión Social $(\alpha=.72)$; Violencia a través de las TIC $(\alpha=.89)$; Violencia Verbal del Alumnado hacia el Profesorado $(\alpha=.75)$; y Violencia del Profesorado hacia el Alumnado $(\alpha=.89)$.

\section{PROCEDIMIENTO}

El alumnado fue seleccionado mediante un muestreo aleatorio estratificado por racimo, estableciendo la parte proporcional de la muestra. La población de la que fue extraído fue la totalidad de estudiantes que asisten a centros educativos sostenidos con fondos públicos (públicos y concertados) en los que se imparte Educación Secundaria Obligatoria (ESO) en Galicia. Se seleccionaron centros y, en cada centro seleccionado, se aplicó la encuesta al conjunto del alumnado de ESO. Una vez determinada la muestra, se contactó con los equipos directivos de los centros, con el objeto de explicarles la finalidad y alcance de la investigación y solicitar su autorización para poder aplicar el cuestionario, estableciendo un calendario de aplicación en horas de tutoría. Paralelamente, se informó a los padres sobre la investigación, obteniéndose su consentimiento acerca de la participación de sus hijos en el estudio. La recogida de datos se llevó a cabo durante el curso 2014-2015. Antes de contestar al cuestionario, los estudiantes 
fueron informados de la finalidad del estudio y su carácter confidencial, solicitándoles su participación voluntaria y anónima. Las instrucciones explicativas fueron las mismas en todas las aulas y por el mismo equipo investigador, a fin de evitar un factor de sesgo.

\section{ANÁLISIS DE DATOS}

Se analizó la frecuencia de cada de tipo de violencia escolar percibida por el alumnado calculando las medias y porcentajes de respuesta a cada opción de la escala en cada ítem. Los análisis fueron realizados con el paquete estadístico SPSS 21.0 (IBM Corp., 2012).

\section{RESULTADOS}

El nivel de violenciaescolar percibido para cada tipo e indicador específico de violencia escolar se muestra en las tablas 2,3 y 4 . Atendiendo a estos resultados, se observa que el tipo de violencia escolar percibido como más habitual por parte del alumnado de ESO de Galicia es la disrupción en el aula. El segundo tipo de violencia escolar más habitual es la violencia verbal entre estudiantes. En concreto, la violencia verbal indirecta (hablar mal a las espaldas, rumores,..) se percibe más habitual que la directa (insultos a la cara). El tercer tipo de violencia escolar percibido como más habitual por los estudiantes es la violencia de profesorado hacia alumnado, especialmente el uso inadecuado de castigos y la preferencia o manía por ciertos alumnos.
En cuarto lugar, la violencia verbal de alumnado a profesorado. Los malos modales y las faltas de respeto son más habituales que los insultos directos. En quinto lugar, la violencia física directa y amenazas entre estudiantes. No obstante, el uso de navajas u otros objetos peligrosos para amenazar se encuentra entre los tipos de violencia menos habituales. En sexto lugar, la exclusión social. La exclusión por buenos resultados académicos es más habitual que por bajo rendimiento. En séptimo lugar, la violencia física indirecta por parte del alumnado. La dirigida al profesorado (desperfectos intencionados, esconder cosas, robos) se encuentra entre los tipos de violencia menos habituales. Es menos habitual que los robos entre estudiantes o de objetos del centro educativo.

Por último, la violencia a través de las TIC es, globalmente considerada, menos habitual que el resto de los tipos de violencia analizados. No obstante, algunos indicadores específicos, como los comentarios ofensivos, insultantes o amenazantes en las redes sociales entre estudiantes presentan una frecuencia significativa. Los comentarios ofensivos en las redes sociales son más habituales entre estudiantes que de alumnado hacia profesorado. La violencia verbal indirecta (comentarios en redes sociales) es más habitual que la directa (mensajes al móvil de la víctima). La violencia verbal a través de las TIC es más habitual que el uso de imágenes (hacer o difundir grabaciones o fotografías ofensivas).

\begin{tabular}{|c|c|c|c|}
\hline \multicolumn{4}{|c|}{$\begin{array}{c}\text { Tabla } 2 . \\
\text { Nivel de violencia escolar percibida por el alumnado según los factores. }\end{array}$} \\
\hline Orden & Factor & $M$ & D.T. \\
\hline $1^{\circ}$ & Disrupción en el aula (DA) & 3.29 & .92 \\
\hline $2^{\circ}$ & Violencia verbal del alumnado hacia alumnado (WAA) & 2.90 & .89 \\
\hline $3^{\circ}$ & Violencia del profesorado hacia el alumnado (NPA) & 2.31 & .85 \\
\hline $4^{\circ}$ & Violencia verbal del alumnado hacia el profesorado (WAP) & 2.27 & .85 \\
\hline $5^{\circ}$ & Violencia física directa y amenazas entre estudiantes (VFDAE) & 2.00 & .80 \\
\hline $6^{\circ}$ & Exclusión social (ES) & 1.86 & .80 \\
\hline $7^{\circ}$ & Violencia física indirecta por parte del alumnado (VFIA) & 1.78 & .71 \\
\hline $8^{\circ}$ & Violencia a través de las TIC (NTIC) & 1.75 & .70 \\
\hline
\end{tabular}


Tabla 3

Descriptivos de los ítems del CUVE3-ESO con el total de la muestra de ESO $(N=4943)$, por orden de frecuencia (puestos $1^{\circ}$ a $\left.25^{\circ}\right)$.

\begin{tabular}{|c|c|c|c|c|c|c|c|c|c|c|}
\hline \multirow[b]{2}{*}{ Orden } & \multirow{2}{*}{ Ítem } & \multirow[b]{2}{*}{ Factor } & \multirow[b]{2}{*}{ Enunciado } & \multirow[b]{2}{*}{$M$} & \multirow[b]{2}{*}{ D.T. } & \multicolumn{5}{|c|}{ Escala (\%) } \\
\hline & & & & & & 1 & 2 & 3 & 4 & 5 \\
\hline $1^{\circ}$ & 30 & DA & Hay alumnado que ni trabaja ni deja trabajar al resto. & 3.38 & 1.26 & 7.3 & 20.4 & 24.5 & 22.4 & 25.4 \\
\hline $2^{\circ}$ & 43 & DA & $\begin{array}{l}\text { El alumnado dificulta las explicaciones del profesorado hablando } \\
\text { durante la clase. }\end{array}$ & 3.35 & 1.21 & 6.9 & 19.8 & 26.7 & 25.0 & 21.6 \\
\hline $3^{\circ}$ & 17 & DA & $\begin{array}{l}\text { El alumnado dificulta las explicaciones del profesor o profesora } \\
\text { con su comportamiento durante la clase. }\end{array}$ & 3.14 & 1.17 & 7.8 & 24.0 & 29.2 & 24.3 & 14.7 \\
\hline $4^{\circ}$ & 11 & WAA & Los estudiantes hablan mal unos de otros. & 3.12 & 1.24 & 8.7 & 27.1 & 26.3 & 19.4 & 18.5 \\
\hline $5^{\circ}$ & 6 & WAA & $\begin{array}{l}\text { Hay estudiantes que extienden rumores negativos acerca de } \\
\text { compañeros y compañeras. }\end{array}$ & 2.86 & 1.18 & 12.8 & 28.7 & 28.3 & 19.9 & 10.3 \\
\hline $6^{\circ}$ & 1 & WAA & $\begin{array}{l}\text { El alumnado pone motes molestos a sus compañeros o } \\
\text { compañeras. }\end{array}$ & 2.86 & 1.08 & 9.8 & 28.3 & 37.0 & 16.5 & 8.5 \\
\hline $7^{\circ}$ & 25 & VPA & $\begin{array}{c}\text { El profesorado baja la nota a algún alumno o alumna como } \\
\text { castigo. }\end{array}$ & 2.81 & 1.31 & 18.6 & 26.9 & 23.4 & 17.2 & 13.9 \\
\hline $8^{\circ}$ & 2 & VPA & El profesorado tiene preferencias por ciertos alumnos o alumnas. & 2.80 & 1.29 & 17.9 & 27.4 & 25.2 & 15.6 & 13.9 \\
\hline $9 \circ$ & 24 & WAA & El alumnado insulta a sus compañeros o compañeras. & 2.75 & 1.21 & 14.7 & 33.7 & 25.0 & 15.1 & 11.5 \\
\hline $10^{\circ}$ & 9 & VPA & El profesorado tiene manía a algunos alumnos o alumnas. & 2.69 & 1.30 & 21.4 & 28.1 & 23.1 & 15.2 & 12.2 \\
\hline $11^{\circ}$ & 21 & VPA & El profesorado castiga injustamente. & 2.65 & 1.24 & 19.5 & 31.4 & 23.8 & 14.8 & 10.4 \\
\hline $12^{\circ}$ & 4 & WAP & El alumnado habla con malos modales al profesorado. & 2.51 & 1.02 & 15.7 & 37.6 & 30.6 & 12.1 & 3.9 \\
\hline $13^{\circ}$ & 18 & VPA & El profesorado ignora a ciertos alumnos o alumnas. & 2.45 & 1.28 & 28.3 & 29.3 & 20.7 & 12.2 & 9.5 \\
\hline $14^{\circ}$ & 39 & VPA & El profesorado no escucha a su alumnado. & 2.33 & 1.21 & 29.4 & 33.4 & 19.4 & 10.5 & 7.3 \\
\hline $15^{\circ}$ & 16 & WAP & El alumnado falta al respeto a su profesorado en el aula. & 2.30 & 1.03 & 23.5 & 39.9 & 23.4 & 10.0 & 3.2 \\
\hline $16^{\circ}$ & 12 & VTIC & $\begin{array}{l}\text { Hay estudiantes que publican comentarios en Tuenti, Facebook... } \\
\text { de ofensa, insulto o amenaza acerca de compañeros/as. }\end{array}$ & 2.24 & 1.20 & 33.8 & 31.6 & 17.6 & 11.2 & 5.8 \\
\hline $17^{\circ}$ & 28 & VFDAE & $\begin{array}{l}\text { Determinados estudiantes pegan a compañeros o compañeras } \\
\text { dentro del recinto escolar. }\end{array}$ & 2.21 & 1.12 & 30.3 & 37.8 & 17.8 & 9.0 & 5.1 \\
\hline $18^{\circ}$ & 8 & VFIA & $\begin{array}{l}\text { Ciertos estudiantes roban objetos o dinero de otros compañeros o } \\
\text { compañeras. }\end{array}$ & 2.19 & 1.13 & 32.6 & 34.4 & 18.6 & 9.8 & 4.6 \\
\hline $19^{\circ}$ & 40 & VFDAE & El alumnado protagoniza peleas dentro del recinto escolar. & 2.12 & 1.06 & 32.6 & 37.5 & 18.6 & 7.8 & 3.5 \\
\hline $20^{\circ}$ & 38 & VFDAE & $\begin{array}{l}\text { Algunos alumnos o alumnas protagonizan agresiones físicas en las } \\
\text { cercanías del recinto escolar. }\end{array}$ & 2.11 & 1.12 & 36.4 & 33.1 & 17.7 & 8.3 & 4.5 \\
\hline $21^{\circ}$ & 15 & VFDAE & $\begin{array}{l}\text { Los estudiantes amenazan a otros de palabra para meterles miedo } \\
\text { u obligarles a hacer cosas. }\end{array}$ & 2.11 & 1.11 & 36.5 & 33.2 & 17.4 & 9.1 & 3.8 \\
\hline
\end{tabular}


Tabla 3. (Continuación)

Descriptivos de los ítems del CUVE3-ESO con el total de la muestra de ESO $(\mathrm{N}=4943)$, por orden de frecuencia $\left(p u e s t o s 1^{\circ}\right.$ a $\left.25^{\circ}\right)$.

\begin{tabular}{|c|c|c|c|c|c|c|c|c|c|c|}
\hline $22^{\circ}$ & 5 & VFIA & Ciertos estudiantes roban objetos o dinero del centro educativo. & 2.09 & 1.12 & 38.1 & 32.2 & 16.9 & 8.7 & 4.1 \\
\hline $23^{\circ}$ & 7 & ES & $\begin{array}{l}\text { Algunos estudiantes son discriminados por sus compañeros o } \\
\text { compañeras por sus buenos resultados académicos. }\end{array}$ & 2.01 & 1.14 & 43.6 & 28.7 & 15.2 & 8.4 & 4.1 \\
\hline $24^{\circ}$ & 20 & WAP & Los estudiantes insultan a profesores o profesoras. & 1.99 & 1.09 & 40.7 & 33.6 & 15.4 & 6.2 & 4.1 \\
\hline $25^{\circ}$ & 14 & VPA & El profesorado ridiculiza al alumnado. & 1.99 & 1.12 & 43.7 & 29.6 & 16.0 & 6.1 & 4.7 \\
\hline $\begin{array}{l}\text { DA }= \\
\text { WAP } \\
\text { Social; } \\
\text { Valores }\end{array}$ & & $\begin{array}{l}\text { Aula } \\
\text { al de } \\
\text { ia } a \\
=\mathrm{N}\end{array}$ & $\begin{array}{l}\text { AA = Violencia Verbal de Alumnado hacia Alumnado } \\
\text { nnado hacia Profesorado; VFDAE = Violencia Física } \\
\text { s de las TIC; VFIA = Violencia Física Indirecta por par } \\
\text {; } 2=\text { Pocas veces; } 3=\text { Algunas veces; } 4=\text { Muchas }\end{array}$ & $\begin{array}{l}A= \\
\text { cta } y \\
\text { el } A / \\
5\end{array}$ & nad & entr & tudic & $s ; \mathrm{ES}$ & Exc & \\
\hline
\end{tabular}

Tabla 4

Descriptivos de los ítems del CUVE3-ESO con el total de la muestra de ESO (N = 4943), por orden de frecuencia (puestos $26^{\circ}$ a $44^{\circ}$ ).

\begin{tabular}{|c|c|c|c|c|c|c|c|c|c|c|}
\hline \multirow[b]{2}{*}{ Orden } & \multirow[b]{2}{*}{ Ítem } & \multirow[b]{2}{*}{ Factor } & \multirow[b]{2}{*}{ Enunciado } & \multirow[b]{2}{*}{$M$} & \multirow[b]{2}{*}{ D.T. } & \multicolumn{5}{|c|}{ Escala (\%) } \\
\hline & & & & & & 1 & 2 & 3 & 4 & 5 \\
\hline $26^{\circ}$ & 33 & VPA & Hay profesores y profesoras que insultan al alumnado. & 1.98 & 1.13 & 43.3 & 31.5 & 14.1 & 6.3 & 4.9 \\
\hline $27^{\circ}$ & 34 & ES & $\begin{array}{l}\text { Hay estudiantes que son discriminados por compañeros o } \\
\text { compañeras por diferencias culturales, étnicas o religiosas. }\end{array}$ & 1.91 & 1.14 & 49.1 & 26.8 & 12.4 & 7.1 & 4.5 \\
\hline $28^{\circ}$ & 36 & VTIC & $\begin{array}{l}\text { Algunos estudiantes envían mensajes a compañeros/as a través } \\
\text { del Tuenti, Facebook,.. de ofensa, insulto o amenaza. }\end{array}$ & 1.87 & 1.06 & 47.6 & 30.0 & 13.2 & 6.0 & 3.2 \\
\hline 290 & 42 & ES & $\begin{array}{l}\text { Algunos estudiantes son discriminados por sus compañeros o } \\
\text { compañeras por su nacionalidad. }\end{array}$ & 1.84 & 1.07 & 50.6 & 27.8 & 12.3 & 5.7 & 3.5 \\
\hline $30^{\circ}$ & 10 & VTIC & $\begin{array}{l}\text { Algunos estudiantes graban o hacen fotos a compañeros o } \\
\text { compañeras con el móvil, para burlarse. }\end{array}$ & 1.82 & 1.03 & 50.0 & 29.0 & 12.7 & 5.7 & 2.6 \\
\hline $31^{\circ}$ & 3 & VTIC & $\begin{array}{l}\text { Hay estudiantes que envían a compañeros/as mensajes a través } \\
\text { del correo electrónico, de ofensa, insulto o amenaza. }\end{array}$ & 1.81 & 1.01 & 49.8 & 29.5 & 12.7 & 5.7 & 2.3 \\
\hline $32^{\circ}$ & 29 & VPA & $\begin{array}{c}\text { Ciertos profesores o profesoras intimidan o atemorizan a algún } \\
\text { alumno o alumna. }\end{array}$ & 1.77 & 1.08 & 55.4 & 24.6 & 11.2 & 4.8 & 4.0 \\
\hline $33^{\circ}$ & 32 & VTIC & $\begin{array}{l}\text { Ciertos estudiantes publican comentarios en Tuenti, Facebook... de } \\
\text { ofensa, insulto o amenaza a profesores o profesoras. }\end{array}$ & 1.77 & 1.04 & 53.6 & 27.1 & 10.9 & 5.4 & 3.0 \\
\hline $34^{\circ}$ & 13 & ES & $\begin{array}{l}\text { Determinados estudiantes son discriminados por sus compañeros o } \\
\text { compañeras por sus bajas notas. }\end{array}$ & 1.69 & .98 & 56.8 & 27.0 & 9.4 & 4.1 & 2.7 \\
\hline $35^{\circ}$ & 31 & VFIA & $\begin{array}{l}\text { Determinados estudiantes causan desperfectos } \\
\text { intencionadamente en pertenencias del profesorado. }\end{array}$ & 1.69 & .98 & 57.1 & 26.3 & 10.2 & 4.1 & 2.4 \\
\hline $36^{\circ}$ & 27 & VTIC & $\begin{array}{c}\text { Hay estudiantes que graban o hacen fotos a profesores o } \\
\text { profesoras con el móvil, para burlarse. }\end{array}$ & 1.68 & .97 & 57.1 & 25.9 & 10.7 & 4.1 & 2.2 \\
\hline $37^{\circ}$ & 41 & VTIC & $\begin{array}{l}\text { Los estudiantes publican en Internet fotos o vídeos ofensivos de } \\
\text { compañeros o compañeras. }\end{array}$ & 1.66 & .94 & 57.2 & 27.2 & 10.0 & 3.6 & 2.1 \\
\hline $38^{\circ}$ & 19 & VTIC & $\begin{array}{l}\text { Ciertos estudiantes envían a compañeros o compañeras mensajes } \\
\text { con el móvil de ofensa, insulto o amenaza. }\end{array}$ & 1.65 & .89 & 56.0 & 29.4 & 9.7 & 3.5 & 1.4 \\
\hline 390 & 37 & VPA & El profesorado amenaza a algún alumno o alumna. & 1.60 & .98 & 64.0 & 21.5 & 8.5 & 3.0 & 3.1 \\
\hline
\end{tabular}


Tabla 4. (Continuación)

Descriptivos de los ítems del CUVE3-ESO con el total de la muestra de ESO $(N=4943)$, por orden de frecuencia (puestos $26^{\circ}$ a $44^{\circ}$ ).

\begin{tabular}{|c|c|c|c|c|c|c|c|c|c|c|}
\hline $40^{\circ}$ & 22 & VFIA & $\begin{array}{l}\text { Algunos estudiantes esconden pertenencias o material del } \\
\text { profesorado, para molestarle deliberadamente. }\end{array}$ & 1.52 & .91 & 67.9 & 19.5 & 7.3 & 3.5 & 1.8 \\
\hline $41^{\circ}$ & 44 & VIIC & $\begin{array}{l}\text { Los estudiantes publican en Internet fotos o vídeos ofensivos de } \\
\text { profesores o profesoras. }\end{array}$ & 1.51 & .90 & 67.2 & 21.1 & 6.8 & 2.8 & 2.1 \\
\hline $42^{\circ}$ & 23 & VIIC & $\begin{array}{l}\text { Hay estudiantes que graban o hacen fotos a compañeros o } \\
\text { compañeras con el móvil, para amenazarles o chantajearles. }\end{array}$ & 1.49 & .84 & 66.9 & 22.1 & 7.2 & 2.4 & 1.4 \\
\hline $43^{\circ}$ & 35 & VFDAE & $\begin{array}{l}\text { Algunos estudiantes amenazan a otros/as con navajas u otros } \\
\text { objetos para meterles miedo u obligarles a hacer cosas. }\end{array}$ & 1.44 & .86 & 72.7 & 16.9 & 6.2 & 2.3 & 1.9 \\
\hline $44^{\circ}$ & 26 & VFIA & Algunos estudiantes roban cosas del profesorado. & 1.43 & .80 & 71.3 & 19.4 & 6.1 & 1.7 & 1.5 \\
\hline
\end{tabular}

\section{DISCUSIÓN}

El presente estudio ha analizado la violencia escolar según la opinión del alumnado, aportando información relevante para el autodiagnóstico de los centros de enseñanza de educación secundaria obligatoria. El objetivo consistía en conocer las formas de violencia escolar que perciben los adolescentes en sus centros educativos, es decir, aquellas conductas intencionadas mediante las cuales se causa daño, vividas directamente o conocidas indirectamente.

Así pues, en primer lugar, se ha puesto de manifiesto que la violencia escolar entre el alumnado de educación secundaria obligatoria cuenta con niveles bajos, en la línea de otras investigaciones (Guerra, ÁlvarezGarcía, Núñez, Castro y Vargas, 2011 ; Martín y Villegas, 2016). Asimismo, puede observarse que las diferentes formas de violencia escolar sí aparecen en mayor o menor medida, y aunque los porcentajes son reducidos, sí presentan oscilaciones importantes según el tipo de violencia (Cerezo, 2009; Perren, Dooley, Shaw y Cross, 2010; Sánchez-Lacasa y Cerezo, 2010).

Respecto a las formas de violencia escolar, en términos globales predomina la disrupción en el aula (no dejar trabajar y dificultar las explicaciones en el aula), la violencia verbal (rumores, motes e insultos), y la violencia del profesorado hacia el alumnado (bajar notas, preferencias, manías, castigos, ignorar o no escuchar al alumnado). En un nivel intermedio se encuentra la violencia física directa y amenazas entre estudiantes (pegar, pelear o amenazar) y la exclusión social (discriminación por diferencias culturales, étnicas, religiosas, nacionalidad o bajas notas). Por el contrario, se mantienen en unos niveles de menor presencia la violencia física indirecta por parte del alumnado (robo de objetos o dinero, causar desperfectos o esconder pertenencias), y la violencia a través de las TIC (envío de mensajes, grabaciones, fotos, o publicaciones en la red a través del teléfono móvil o internet). Ello es coincidente con los estudios realizados por Muñoz, Carreras y Braza (2004); Badía (2005); Cangas, Gázquez, Pérez, Padilla y Miras, (2007); Gázquez, Cangas, PérezFuentes, Padilla y Cano (2007); Garaigordobil y Oñederra (2010); Guerra et al. (2011); Giorgi, Kaplún y Morás (2012); Buendia, Exposito, Aguadez y Sánchez (2015). De hecho, los comportamientos disruptivos son uno de los problemas de convivencia que más preocupa en los centros educativos (Ortega, Romera y Del Rey, 2010). 
En base a estos resultados, el haber identificado mayor frecuencia de situaciones de violencia escolar de menor gravedad (disrupción, insultos, bajar notas, preferencias) entre el alumado de ESO, no reduce su importancia (Baridón y Martín, 2014), ya que su repetitividad eleva esta problemática hacia patrones de conducta más graves relacionados con la violencia física. Asimismo, la violencia ejercida a través de las nuevas tecnologías de la información y comunicación es la de menor presencia en los centros de ESO, se mantiene en porcentajes muy bajos similares a los obtenidos en otras investigaciones (Defensor del Pueblo, 2007; Smith, Mahdavi, Carvalho y Tippet, 2006; Slonje y Smith, 2008; Ybarra y Mitchell, 2004), y difiere de otros estudios que constatan mayor prevalencia entre los adolescentes (Buelga, Cava y Musitu, 2010; Estévez, Villardón, Calvete, Padilla y Orue, 2010; Jiménez y García, 2010; Ortega, Calmaestra y Mora, 2008). Tal vez, aunque se hayan llevado a cabo intervenciones contra este tipo de violencia (Garaigordobil y Martínez-Valderrey, 2015; Ortega, Del Rey y Casas, 2016), aún se observa un cierto vacío por tratarse de un fenómeno relativamente reciente.

Finalmente, los resultados de nuestro trabajo confirman la importancia de conocer las formas de violencia escolar con mayor presencia en los centros de educación secundaria obligatoria, para aportar soluciones a los nuevos retos derivados de la creciente conflictividad en las escuelas y aulas. De ahí, la relevancia de este estudio como eje de diagnóstico sobre las formas de violencia, para lograr acciones de calidad en su prevención e intervención (Buelga, Musitu, Vera, Ávila y Arango, 2009; Cooley-Strickland, Griffin y Furr-Holden, 2011 ). No obstante, la investigación presenta ciertas limitaciones, entre las que destaca mencionar su transversalidad (los datos pueden verse afectados por el paso del tiempo), y los efectos de deseabilidad social (autoinformes). Por ello, sería fundamental la realización de futuros estudios longitudinales utilizando técnicas de corte cualitativo para lograr una mejor profundización en esta temática.

\section{Conflicto de intereses}

Los autores declaran no tener ningún conflicto de intereses.

\section{REFERENCIAS}

Almeida, A., Lisboa, C., \& Caurcel, M. J. (2007). ¿Por qué ocurren los malos tratos entre iguales? Explicaciones causales de adolescentes portugueses y brasileños. Revista Interamericana de Psicología/ Interamerican Journal of Psychology, 41(2), 107. 118.

Álvarez-García, D., Dobarro, A., Alvarez, L., Núñez, J. C., \& Rodríguez, C. (2014). La violencia escolar en los centros de educacion secundaria de Asturias desde la perspectiva del alumnado. Educacion XX1, 17(2), 337-360, doi: 10.5944/educxx1.17.2.11494

Álvarez-García, D., Núñez, J. C., \& Dobarro, A. (2012). CUVE3. Cuestionario de Violencia Escolar3. Barakaldo: ALBOR-COHS.

Álvarez-García, D., Rodríguez, C., Gonzalez-Castro, P., Núñez, J. C., \& Álvarez, L. (2010). La formacion de los futuros docentes frente a la violencia escolar. Revista de Psicodidactica, 15(1), 35-56.

Arias, L. (2009). Una mirada al fenómeno de la violencia escolar en Costa Rica. Revista Electrónica Educare, XI/(1), 41-51.

Ayala-Carrillo, M. R. (2015). Violencia escolar: Un problema complejo. Ra Ximhai, $17(4), 493-509$.

Badía, M. M. (2005). Gravedad e importancia de los comportamientos de indisciplina. Causas y factores asociados a ciertas conductas: una revisión de diferentes estudios. Psicología Educativa, 2(11), 65-78.

Baridon, D., \& Martín, G. (2014). Violencia escolar en estudiantes de Educación Media. Ciencias Psicológicas, 8(2), 173-183.

Berger, Ch., \& Lisboa, C. (2009). Violencia escolar. Estudios y posibilidades de intervención en Latinoamérica. Santiago de Chile: Editorial Universitaria.

Buelga, S., Cava, M. J., \& Musitu, G. (2010). Cyberbullying: victimización entre adolescentes a través del teléfono móvil y de Internet. Psicothema, 22, 784-789.

Buelga, S., Musitu, G., Vera, A., Ávila, M. E., \& Arango, C. (2009). Psicología Social Comunitaria. México: Trillas.

Buendia, L., Exposito, J., Aguadez, E. M., \& Sánchez, Ch.A. (2015). Análisis de la convivencia escolar en las aulas multiculturales de Educación Secundaria. Revista de Investigación Educativa, 33(2), 303-319, doi: 10.6018/rie.33.2.211491

Cabezas, H., \& Monge, M. (2013). Violencia escolar, un problema que aumenta en la escuela primaria costarricense. Revista Electrónica Actualidades Investigativas en Educación, 13(2), 1-20.

Cajigas, N., Luzardo, M., \& Mungay, M. (2013). Una mirada psicológica de la violencia en los centros educativos. Prevalencia de afectos, actitudes y conductas negativas de adolescentes del secundario público en Montevideo, Uruguay. Deutschland, Alemania: Académica Española. 
Cangas, A. J., Gázquez, J. J., Pérez, M. C., Padilla, D., \& Miras, F. (2007). Evaluación de la violencia escolar y su afectación personal en una muestra de estudiantes europeos. Psicothema, 19, 114-119.

Cava, M. J., Buelga, S., Musitu, G., \& Murgui, S. (2010). Violencia escolar entre adolescentes y sus implicaciones en el ajuste psicosocial: un estudio longitudinal. Revista de Psicodidáctica, 15(1), 21 -34.

Cerezo, F. (2009). Bullying: Análisis de la situación en las aulas españolas. International Journal of Psychology and Psychological Therapy, 9(3), 367. 378.

Cerezo, F., \& Calvo, A. R. (2011). Programa CIP: intervención psicoeducativa y tratamiento diferenciado del bullying. Concienciar, informar, prevenir. Madrid: Pirámide.

Cooley-Strickland, Q., Griffin, S. B., \& Furr-Holden, D. (2011). Efectos de la exposición de los adolescentes a la violencia en la comunidad: el Proyecto MORE. Psychosocial Intervention, 20, 131-148.

Defensor del Pueblo (2007). Violencia escolar: el maltrato entre iguales en la Educación Secundaria Obligatoria (1999-2006). Madrid: Publicaciones del Defensor del Pueblo.

Díaz-Aguado, M. J., Martínez, R., \& Martín, G. (2004). Prevención de la violencia y lucha contra la exclusión desde la adolescencia. Madrid: Ministerio de Trabajo y Asuntos Sociales, INJUVE.

Espelage, D. L., \& Swearer, S. M. (2010). A socialecological model for bullying prevention and intervention: Understanding the impact of adults on the social ecology of youngsters. En S. R. Jimenson, S. S. Swearer \& D. L. Espelage (Eds.), Handbook of bullying in schools: An international perspective (pp. 61-86). Nueva York: Routledge.

Estévez, A., Villardón, L., Calvete, E., Padilla, P., \& Orue, I. (2010). Adolescentes víctimas de cyberbullying: prevalencia y características. Revista de Psicología Clínica y de la Salud, 18, 73-89.

Félix, V., Soriano, M., \& Godoy, C. (2009). Un estudio descriptivo sobre el acoso y violencia escolar en la educación obligatoria. Escritos de Psicología, 2(2), 43-51.

Garaigordobil, M. (2009). Intervención socioeducativa para promover la conducta prosocial y prevenir la violencia. Organización y gestión educativa: Revista del Fórum Europeo de Administradores de la Educación, 17(3), 31 -32.

Garaigordobil, M., \& Martinez-Valderrey, V. (2015). Effects of Cyberprogram 2.0 on "face-to-face" bullying, cyberbullying and empathy. Psicothema, 27(1), 45-51, doi:10.7334/psicothema2014.78

Garaigordobil, M., Martínez-Valderrey, V., \& Machimbarrena, J. M. (2017). Intervención en el bullying y cyberbullying: Evaluación del caso Martín. Revista de Psicología Clínica con Niños y Adolescentes, 4(1), 25-32.

Garaigordobil, M., \& Oñederra, J. A (2010). Los centros educativos ante el acoso escolar: actuaciones del profesorado, acciones sancionadoras y actividades de prevención. Informació Psicológica, 99, 4-18.
García, R. (2011). La violencia escolar. Educainnova Magazine, 13(1), 40-44.

Gázquez, J. J., Cangas, A. J., Pérez-Fuentes, M. C., Padilla, D., \& Cano, A. (2007). Percepción de la violencia escolar por parte de los familiares: un estudio comparativo en cuatro países europeos. International Journal of Clinical and Health Psychology, 7, 93-105.

Giorgi, V., Kaplún, G., \& Morás, L. E. (2012). La violencia está en los otros. La palabra de los actores educativos. Montevideo: Trilce.

Guerra, C., Alvarez-García, D., Dobarro, A., Nuñez, J. C., Castro, L., \& Vargas, J. (2011). Violencia Escolar en Estudiantes de Educación Secundaria de Valparaíso (Chile): Comparación con una muestra Española. Revista Iberoamericana de Psicología y Salud, 2(1), 75-98.

Hernández, I., Martínez, C., \& Torres, A. (2006). Percepción de la violencia escolar por parte de las personas responsables de la dirección de los centros de enseñanza de Alicante: un estudio cualitativo. Revista Española de Salud Pública, 80(4), 387-394.

IBM Corp. (2012). SPSS Statistics for Windows, Version 21.0. Armonk, NY: IBM Corp.

Jiménez, A., \& García, A. (2010). Cyberbullying: un estudio descriptivo. En J. J. Gázquez y M.C. Pérez (Eds.), Investigación en convivencia escolar: variables relacionadas (pp. 137-141). Granada: GEU.

Martín, M., \& Villegas, J. M. (2016). Clima, autoestima y violencia escolar en estudiantes de séptimo básico a cuarto medio en una escuela de la comuna de Alto Hospicio, Chile. Huellas, Revista del Observatorio sobre la Violencia y Convivencia en la Escuela, 2(4), 60-74.

Martínez, C., Hernández-Aguado, I., \& Torres, A. M. (2006). Percepción de la violencia escolar por parte de las personas responsables de la dirección de los centros de enseñanza de Alicante: Un estudio cualitativo. Revista Española de Salud Pública, 80(4), 387-394.

Martínez, J. W., Tovar, J. R., Rojas., C., \& Duque-Franco, A. (2008). Agresividad en los escolares y su relación con las normas familiares. Revista Colombiana de Psiquiatría, 37(3), 365-377.

Muñoz, J. M., Carreras, M. R., \& Braza, P. (2004). Aproximaciones a las actividades y estrategias del pensamiento social y su relación con los comportamientos disruptivos en el aula de la educación secundaria. Murcia, España: Servicios de Publicaciones de la Universidad de Murcia.

Muñoz, M. C., Pérez, D. \& Martín, E. (2006). ¿Qué penalizan los docentes?: análisis de la disciplina a través de los partes de incidencia. Infancia y Aprendizaje, 29, 423-435.

Musri, S. (2012). Acoso escolar y estrategias de prevención en educación escolar básica y nivel medio. Tesis inédita de maestría. San Lorenzo (Paraguay): Universidad Tecnológica Intercontinental.

Ortega, R., Calmaestra, J., \& Mora, J. (2008) Cyberbullying. Internacional Journal of Psychology and Psychological Therapy, 8(2), 183-192. 
Ortega, R., Del Rey, R., \& Casas, J. A. (2016). Evaluar el bullying y el cyberbullying validación española del EBIP-Q y del ECIP-Q. Psicología Educativa, 22, 71 79.

Ortega, R., Romera, E., \& Del Rey, R. (2010). Construir la convivencia escolar: Un modelo para la prevención de la violencia, la competencia social y la educación ciudadana. En A.M. Foxley (Ed.), Aprendiendo a vivir juntos (pp 23-49). Santiago: LOM.

Osorio, F. (2006). Violencia en las escuelas. Un análisis desde la subjetividad. Buenos Aires: Centro de Publicaciones Educativas y Material Didáctico.

Pagani, L., Tremblay, R. E., Nagin, D., Zoccolillo, M., Vitaro, F., \& McDuff, P. (2009). Risk factor models for adolescent verbal and physical aggression toward fathers. Journal of Family Violence, 24, 173-182.

Palmero, F., Guerrero, C., Gómez, C., Carpi, A., \& Gorayeb, R. (2010). Manual de Teorías Emocionales y Motivacionales. Castellón: Sapientia.

Perren, S., Dooley, J. J., Shaw, T., \& Cross, D. (2010). Bullying in school and cyberspace: Associations with depressive symptoms in Swiss and Australian adolescents. Child and Adolescent Psychiatry and Mental Health, 4(28), 1-10.

Pinheiro, P. S. (2011). Informe Mundial sobre la Violencia contra los niños y las niñas. Ginebra: Naciones Unidas. UNICEF.

Romera, E. M., Del Rey, R., \& Ortega, R. (2011). Factores asociados a la implicación en Bullying: un estudio en Nicaragua. Psychosocial Intervention, 20(2), $161-170$.
Sánchez-Lacasa, C., \& Cerezo, F. (2010). Variables personales y sociales relacionadas con la dinámica bullying en escolares de Educación Primaria. Electronic Journal of Research in Educational Psychology, 8(3), 1015-1032.

Serrano, S. A., \& Iborra, M. I. (2005). Informe violencia entre compañeros en la escuela. Serie Documentos No 9. España: Goaprint, S.L.

Slonge, R., \& Smith, P. (2008). Cyberbullying: another main type of bullying. Scandinavian Journal of Psychology, 49, 147-154.

Smith, P. K., Mahdavi, J., Carvalho, C., \& Tippett, N. (2006). An investigation into cyberbullying, its forms, awareness and impact, and the relationship between age and gender in cyberbullying. London: Anti-Bullying Alliance.

Steffgen, G., Recchia, S., \& Viechtbaver, W. (2013). The link between school climate and violence in school: A metaanalytic review. Aggresion and Violent Behavior, 18(2), 300-309.

Torrego, J. C. (2007). El modelo integrado: un nuevo marco de educativo para la gestión de los conflictos de convivencia desde una perspectiva de centro. Idea La Mancha-Revista de Educación de Castilla-La Mancha, 4, 84-89.

Ybarra, M., \& Mitchell, K. (2004). Youth engaging in online harassment: Associations with caregiver chid relationships, internet use and personal characteristics. Journal of Adolescence, 2, 319-336.
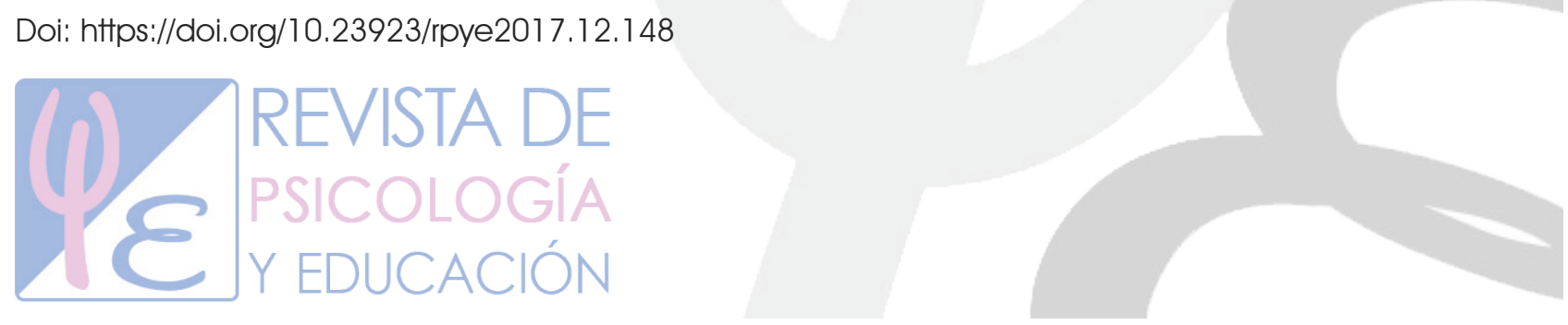